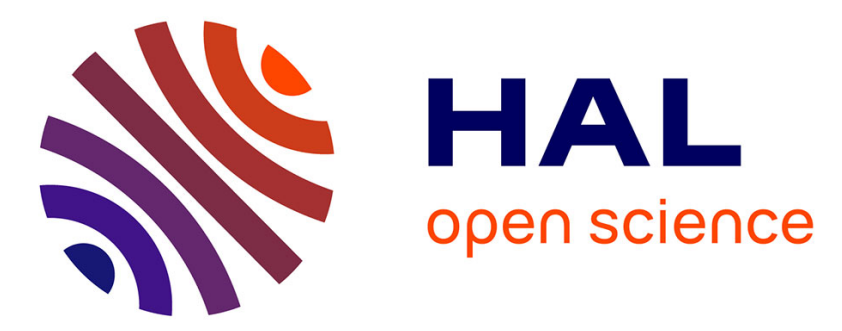

\title{
Transition between tetragonal and hexagonal pattern in binary blends of ABC block copolymers with different chain length
}

Marylène Vayer, Aynur Guliyeva, Fabienne Warmont, Atsushi Takano, Yushu

Matsushita, Christophe Sinturel

\section{To cite this version:}

Marylène Vayer, Aynur Guliyeva, Fabienne Warmont, Atsushi Takano, Yushu Matsushita, et al.. Transition between tetragonal and hexagonal pattern in binary blends of ABC block copolymers with different chain length. European Polymer Journal, 2020, 138, pp.109986. 10.1016/j.eurpolymj.2020.109986 . hal-03181222

\section{HAL Id: hal-03181222 \\ https://hal.science/hal-03181222}

Submitted on 25 Mar 2021

HAL is a multi-disciplinary open access archive for the deposit and dissemination of scientific research documents, whether they are published or not. The documents may come from teaching and research institutions in France or abroad, or from public or private research centers.
L'archive ouverte pluridisciplinaire HAL, est destinée au dépôt et à la diffusion de documents scientifiques de niveau recherche, publiés ou non, émanant des établissements d'enseignement et de recherche français ou étrangers, des laboratoires publics ou privés. 


\section{Transition between tetragonal and hexagonal pattern in binary blends of}

\section{ABC block copolymers with different chain length}

Marylène Vayer $^{1 *}$, Aynur Guliyeva ${ }^{1,2}$, Fabienne Warmont ${ }^{1}$, Atsushi Takano ${ }^{3}$,

Yushu Matsushita ${ }^{3,4}$ and Christophe Sinturel ${ }^{1 *}$

${ }^{1}$ Interfaces, Confinement, Matériaux et Nanostructures (ICMN) UMR 7374, CNRS-Université d’Orléans, CS 40059, F-45071 Orléans, France

${ }^{2}$ Present address: Center for Research in Soft matter \& Polymers, Department of Chemical \& Biomolecular Engineering, University of Delaware, Newark, Delaware 19716, United States

${ }^{3}$ Department of Molecular \& Macromolecular Chemistry, Graduate School of Engineering, Nagoya University, Furo-cho, Chikusa-ku, Nagoya 464-8603, Japan

${ }^{4}$ Present address: Toyota Physical and Chemical Research Institute, Yokomichi 41-1, Nagakute, Aichi 480-1192, Japan

KEYWORDS: Block Copolymer; Order-Order-Transition; Double hexagonal morphology;

Tetragonal morphology; Thin film effect

\section{Corresponding authors}

Christophe Sinturelः christophe.sinturel@univ-orleans.fr

Marylène Vayer: marylene.vayer@univ-orleans.fr 


\title{
Graphical abstract
}

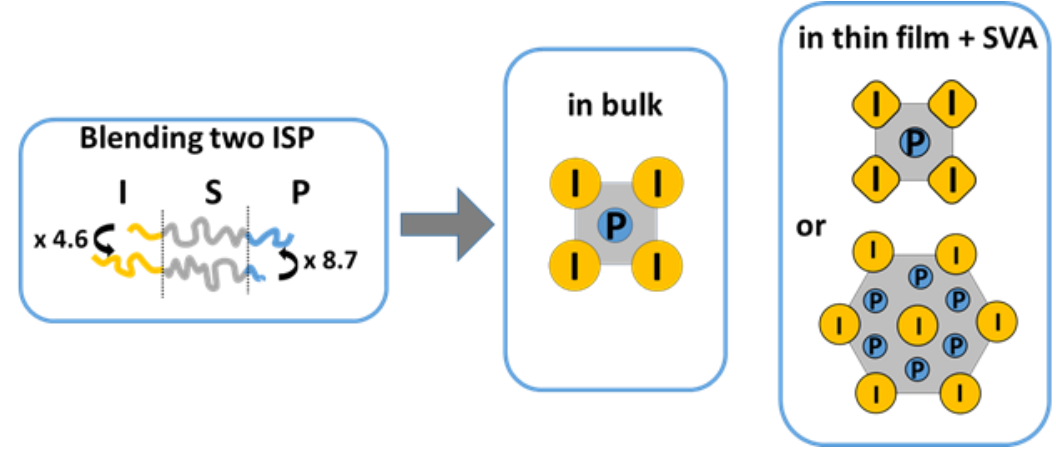

\begin{abstract}
The thin film morphology of binary blends of triblock copolymers (poly(isoprene-blockstyrene-block-(2-vinylpyridine)) ISP after solvent vapor annealing (SVA) was investigated using AFM, GISAXS and TEM. Although bulk samples of binary ISP blends exhibit tetragonal pattern, thin films led either to tetragonal pattern or hexagonal pattern, depending on the annealing time. These transitions were attributed to the formation of non-synchronized wetting layers of I and P, leading to a variation of the inner composition in I and P during SVA. Surprisingly, the hexagonal morphology exhibited centered hexagons of I and surrounding hexagons of $\mathrm{P}$, which is not predicted nor observed for neat block copolymer samples. Although the system must adopt the lowest P/S interface, our results suggest that the effect of chain length with marked difference in the studied blends is playing a major role. Particularly, the presence of long chains of I favors the formation of I domains with bigger size, in order to keep their conformation entropy loss as small as possible.
\end{abstract}




\section{Introduction}

Microphase separation of linear ABC terpolymer has been the object of numerous works [116]. The formed structures and their domains of stabilization depend strongly on the blocks volume fractions and the incompatibility of the blocks with each other ([4-8]). In the case of non-frustrated (i.e. the incompatibility between the end-blocks is higher than the incompatibility between the middle and end blocks) linear polymers, these materials selfassemble into simple ordered structures with spherical, cylindrical, gyroid and lamellar domains. Whereas cylindrical domains are arranged exclusively into a hexagonally packed structure for diblock copolymers, three types of cylindrical morphologies are predicted for triblock terpolymers $[4,5]$.

The first one corresponds to a core-shell version, arranged in hexagonal packing (Figure 1a). In this case, one end-block (e.g. A) is the major component and constitutes the matrix in which core (end-block C)/shell (middle-block B) cylinders are formed. This concerns highly asymmetric non-frustrated linear triblock copolymers $[4,5,9]$.

The second type of morphology corresponds to cylinders consisting in one end-block (e.g. A) arranged in hexagonal packing in a matrix of B, the other end-block (C) being miscible with the middle-block (B) or forming interstitial domains in the matrix (Figure 1b). This concerns triblocks with $\mathrm{B}$ as major component, distinct proportions of $\mathrm{A}$ and $\mathrm{C}$ and intermediate segregation conditions $[4,5]$. For example, Tureau et al. [10, 11] observed for PI-PS-PMMA (ISM) cylinders of I arranged in hexagonal pattern in a matrix of SM.

The last one is the tetragonal structure, with alternating $\mathrm{A}$ and $\mathrm{C}$ cylinders within $\mathrm{B}$ matrix, observed for triblock with middle-block B as the major component and ratio of volume fractions of end-blocks is near unity (Figure 1c) [5,6]. For example, Mogi et al. [12] explored this structure with two kinds of cylinders formed by the two end-blocks for PI-PS-P2VP (ISP) system. 
Beside the structures predicted above, the formation of a double hexagonal structure was repeatedly experimentally reported in the literature, whereas up to date, no arrangement with distinct $\mathrm{A}$ and $\mathrm{B}$ cylinders in a double regular hexagonal structure was predicted to our knowledge (Figure 1d). Brinkmann et al. [13] observed in PS-PB-PMMA (SBM) triblocks having a (0.21:0.57:0.16) (S:B:M) composition, the formation of a new hexagonal morphology with $\mathrm{S}$ and $\mathrm{M}$ cylinders in a matrix of $\mathrm{B}(7 \mathrm{M}$ cylinders forming centered hexagons and $6 \mathrm{~S}$ cylinders forming hexagon around each M cylinder). Such pattern was also described by Asai et al. [14] for ISP system with a composition (I:S:P) (0.12:0.67:0.21), in which P cylindrical domains were arranged in centered hexagons and each $\mathrm{P}$ domain was surrounded by $6 \mathrm{I}$ cylindrical domains. For ISP system, Izumi and al. [15] studied bulk system of binary ISP/S blends, the parent triblocks being all gyroid with composition between (0.13: $0.55: 0.32)$ and (0.34: 0.55 : 0.11$)$. Adding $S$ homopolymer led to blend composition (0.25-0.10: $0.65-0.67$ : 0.08-0.24) and cylindrical structures. I and P were co-arrayed in a tetragonal symmetry up to a critical ratio of $\mathrm{P} / \mathrm{I}$ that depended on the molecular mass of the homopolymer $\mathrm{S}$ (e. g. a ratio of 1 was observed for $\mathrm{Mn}=12 \mathrm{~kg} \cdot \mathrm{mol}^{-1}$ ). Above this ratio, cylinders of I and P were organized within a double hexagonal structure, where $\mathrm{P}$ cylinders form centered hexagons and I block form 6 cylinders surrounding the previous ones. Asai et al. [14] demonstrated that blends of two terpolymers with a selected symmetric composition (volume fraction of S between 0.54 to 0.69 ) lead to alternating rods in a tetragonal structure. Moreover, if end-blocks are composed of a blend of short and long chains, the rods are no longer cylindrical domains but have non-constant mean curvature. $[16,17]$ 


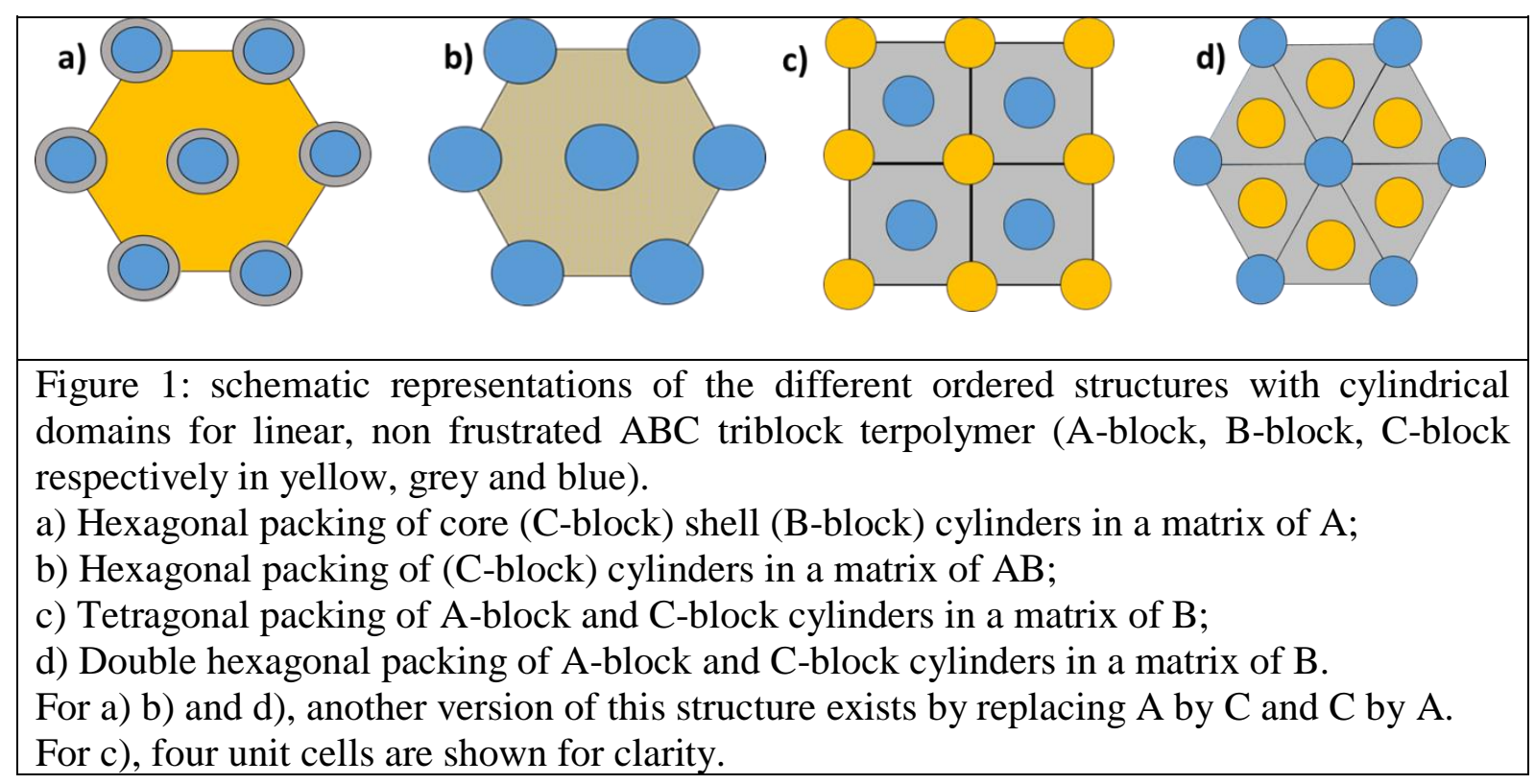

In thin films, the cases are even more complex, since the limited amount of polymer and the enhanced effects of the interfaces lead to stabilization, destabilization and also to orientation of the structures. In addition, solvent swelling, thermodynamics, kinetics make the situation even more complex.[18] In PI-PS-PFS (ISFS) system, Chuang et al. [19] demonstrated that neat copolymer with a (I:S:FS) (0.25:0.65:0.10) exhibits in thin films either square-packed patterns of I and FS cylinders with a composition (0.165:0.67:0.165) or FS-S core-shell cylinders in a hexagonal symmetry in a matrix of I with a composition (0.35:0.51:0.14). Addition of S leads to a (0.16:0.78:0.06) composition in volume and a stabilization of square pattern. In ISP thin films, Guliyeva et al. [20] demonstrated that, for the blend of two ISP triblocks studied by Asai et al. [16] with a symmetric composition (0.20:0.60:0.20), the square pattern is preserved in the inner part of the film under THF vapor annealing, producing rods perpendicular to the surface for short annealing time and rods parallel to the surface for long annealing time. It was shown in this paper that similarly to the bulk, the end-blocks domains are in this case no longer cylinders but rods with flat interface. 
The problematic we address here is the structuration of block polymer blends with near symmetric composition in thin films, with a focus on the possible evolutions from tetragonal to double hexagonal morphology, due to the thin film effect. We also examine the effect of block chains length difference within the blends. For that purpose, we blended two triblocks of ISP with $\mathrm{S}$ as the major block, one with a very long chain of I and the other with a long chain of $\mathrm{P}$. We selected two blends compositions already studied in bulk in previous work by Asai et al. [14] showing tetragonal structures in the bulk.

\section{Materials and methods}

Poly(isoprene-block-styrene-block-(2-vinylpyridine)) triblock terpolymers (ISP) were synthesized by anionic polymerization following a protocol described elsewhere [21]. These ISP X and ISP Y terpolymers have been studied in details by Asai et al. [14] and their molecular and morphological characteristics extracted from this work are presented in table 1. Two binary blends based on ISP X and ISP Y were prepared. The blending characteristics and the corresponding compositions are described in table 2.

\begin{tabular}{|c|c|c|c|c|c|}
\hline & $\mathrm{M}_{\mathrm{n}}$ & $\varphi_{\mathrm{II}}: \varphi_{\mathrm{S}}: \varphi_{\mathrm{P}}$ & \multicolumn{3}{|c|}{ bulk structure } \\
\hline & (1) & (2) & (3) & (4) & (5) \\
\hline ISP X[14] & 223 & $\begin{array}{l}0.12: 0.67: 0.21 \\
357: 1996: 625\end{array}$ & $\begin{array}{l}\mathrm{d}_{\mathrm{cc}}=70 \mathrm{~nm} \\
\mathrm{D}_{\mathrm{I}}=19 \mathrm{~nm} \\
\mathrm{D}_{\mathrm{P}}=32 \mathrm{~nm}\end{array}$ & $P(1)$ & \\
\hline ISP Y[14] & 264 & $\begin{array}{l}0.42: 0.56: 0.02 \\
1649: 2007: 72\end{array}$ & $\begin{aligned} \mathrm{d} & =95 \mathrm{~nm} \\
\mathrm{~L}_{\mathrm{I}} & =48 \mathrm{~nm} \\
\mathrm{~L}_{\mathrm{S}} & =47 \mathrm{~nm} \\
\mathrm{D}_{\mathrm{P}} & =12 \mathrm{~nm}\end{aligned}$ & $\begin{array}{l}P \\
P \\
P \\
P \\
P \\
P \\
P\end{array}$ & \\
\hline
\end{tabular}

Table1. Molecular characteristics of the used block copolymer and their bulk structure. [14]

${ }^{(1)} \mathrm{M}_{\mathrm{n}}$ molecular weight, ${ }^{(2)} \varphi$ volume fraction; $\mathrm{N}$ block length in number of Flory repeating units; ${ }^{(3)} \mathrm{d}_{\mathrm{cc}}$ center to center distance for the cylinders of the centered hexagon; $D$ cylinder diameter. These characteristic lengths are deducted from TEM experiments ${ }^{(4)}$ schematic representation with $\mathrm{I}$ in yellow, $\mathrm{S}$ is grey and $\mathrm{P}$ is blue; ${ }^{(5)}$ representative TEM image, TEM scale bar $=100 \mathrm{~nm}$. 
All the used solvents were purchased from Sigma Aldrich and Roth Sochiel and used as received.

\begin{tabular}{|c|c|c|c|}
\hline $\begin{array}{c}\text { blended } \\
\text { polymers }\end{array}$ & $\begin{array}{c}\text { blending } \\
\text { proportion }(\mathrm{v} / \mathrm{v})\end{array}$ & $\begin{array}{c}\text { volume fraction } \\
(\mathrm{I}: \mathrm{S}: \mathrm{P})\end{array}$ & schematic of block length ratios \\
\hline $\mathrm{X} / \mathrm{Y}$ & $60 / 40$ & $0.26: 0.62: 0.12$ & \\
\hline $\mathrm{X} / \mathrm{Y}$ & $85 / 15$ & $0.17: 0.66: 0.17$ & \\
\hline \multicolumn{3}{|c|}{ Table 2: Blending conditions, volume fraction and blocks length ratios } \\
\hline
\end{tabular}

Solutions of blends were prepared by dissolving polymers in solvent (1,2-dichloroethane) and were stirred for at least 2 hours. $\mathrm{Si}(100)$ substrates (kindly provided by STMicroelectronics Co) of $10 * 10 \mathrm{~mm}^{2}$ were cleaned by sonication in tetrahydrofuran (THF), ethanol and acetone for 10 minutes each. Then the solutions were deposited by spin-coating on Si substrates at $3000 \mathrm{rpm}$ for 30 seconds. The film thicknesses, measured by visible spectral reflectometry using F20 by Filmetrics, ranged from 100 to $120 \mathrm{~nm}$. The accuracy of the determination of the thickness by visible spectral reflectometry was verified on polymer films using Atomic Force Microscopy (AFM) measurement of the depth of a scratch line.

After the deposition, a solvent vapor annealing (SVA) post-treatment was performed. For that purpose, the films were exposed to toluene vapor in a closed glass vessel (1L) containing $3 \mathrm{~mL}$ of solvent at $30^{\circ} \mathrm{C}$ and the structures evolution depending on exposure time was detected.

AFM in the tapping mode was carried out in air with AFM Dimension Icon model (Bruker ICON) and silicon cantilevers RTESP300 from Bruker were used. All the displayed AFM images are height images processed with Gwyddion.

Transmission electron microscopy (TEM) was performed with a Philips CM20 operated at an accelerating voltage of $200 \mathrm{kV}$. For that purpose, the films were removed from the $\mathrm{Si} / \mathrm{SiO}_{2}$ substrate by flotation at the surface of $0.5 \mathrm{M} \mathrm{NaOH}$ solution for more than $5 \mathrm{~h}$. $\mathrm{NaOH}$ attacks the native silica layer and leads to the detachment of film fragments from the substrate. These 
fragments were washed by distilled water to remove $\mathrm{NaOH}$ traces and were transferred onto a TEM grid (for top view analysis) or on a rigid plastic substrate, $1 \mathrm{~mm}$ thick (for sectional view analysis). This plastic substrate was covered by another rigid plastic substrate and the resulting sandwich (with the fragment to be observed in the middle) was cut by ultramicrotome. The obtained sections were transferred onto grids. All the grids were dried at RT for 1 day and were stained. Two staining steps were performed for selectively revealing the domains, namely $\mathrm{OsO}_{4}$ staining for $\mathrm{I}$ and $\mathrm{I}_{2}$ exposure for $\mathrm{P}$ domains. The same staining protocol was carefully followed for all the samples in order to be able to compare the characteristic distances measured on different staining images. These distances were averaged on a minimum of 10 measures and the standard deviation estimated to at most $\pm 3 \mathrm{~nm}$.

GISAXS experiments were performed on the "Xeuss" Xenocs X-ray scattering setup on thin films stained by $\mathrm{I}_{2}$ to increase the electronic density contrast. The two-dimensional GISAXS patterns were recorded using a high sensitivity low noise 2D detection (Pilatus 300K hybrid pixel) placed at a distance of $2329 \mathrm{~mm}$ from the sample, perpendicular to the X-ray beam (parallel beam at $8042 \mathrm{eV}$ ) with a grazing incidence angle of $0.17^{\circ}$ close to the critical angle of the films. The diffraction patterns were recorded, and in-plane GISAXS profiles were extracted.

Surface tensions of blocks and substrate were determined using the Owens Wendt approach. Contact angles of 3 liquids (namely deionized water, diiodomethane and ethylene glycol) were performed using GBX Digidrop apparatus on I, S, P homopolymer films and substrate surfaces.

\section{Results and discussion}

The morphology evolution after toluene vapor exposure was first followed as a function of exposure time using AFM. To address the observed nanostructured patterns, GISAXS, TEM and AFM analyses were combined. 


\section{$\mathrm{X} / \mathrm{Y} \mathbf{8 5 / 1 5}$ blend}

As a function of time, the morphology of the $85 / 15 \mathrm{X} / \mathrm{Y}$ film evolved from disorganized (as spun) to a hexagonal, then square pattern and then disorganized morphology as shown in Figure 2 and SI-1. This last morphology originates from a swelling of the film that allows passing through the order-disorder transition.

\section{X/Y 85/15 blend hexagonal pattern}

Figure 2a displays the AFM image of a surface exhibiting the hexagonal pattern. Despite numerous defects impairing the long-range order, the hexagonal arrangement is evident from this image, at least on a local length scale. The center-to-center distance between holes is evaluated to $72 \mathrm{~nm}$. Figure $2 \mathrm{~b}$ displays a TEM top-view image after successive selective staining by $\mathrm{OsO}_{4}$ and $\mathrm{I}_{2}$. This procedure stains $\mathrm{I}$ domains in black, $\mathrm{P}$ in dark grey and lets $\mathrm{S}$ unstained (light grey). The presence of black dots is due to gold nanoparticles used for other experimental purposes (including tomography, not shown here). On TEM image, round black domains of I adopt a centered hexagonal pattern. Each I domain is surrounded by 6 round dark grey domains of P. This morphology will be designed thereafter by "centered hexagons of I". The center-to-center distance between two I domains is evaluated to $77 \mathrm{~nm}$, the diameters of I and $\mathrm{P}$ domains are evaluated to 27 and $28 \mathrm{~nm}$. The GISAXS pattern obtained on film after $\mathrm{I}_{2}$ staining presents peaks with an extension parallel to the $\mathrm{q}_{\mathrm{z}}$ axis (SI-2a). This is the signature of a homogeneous composition of the film perpendicularly to the substrate in agreement with the TEM-section view image (Figure 2c) which shows rods extending perpendicularly to the surface. The in-plane profile (Figure 2d) presents three main peaks at 0.090, 0.157 and 0.316 $\mathrm{nm}^{-1}$ corresponding to the (10) (11) and (22) diffraction peaks in ratio $\sqrt{ } 3$ and $\sqrt{ } 12$ to the first one of a hexagonal pattern in plane with a cell unit length of $70 \mathrm{~nm}$ in agreement with AFM and TEM results. 


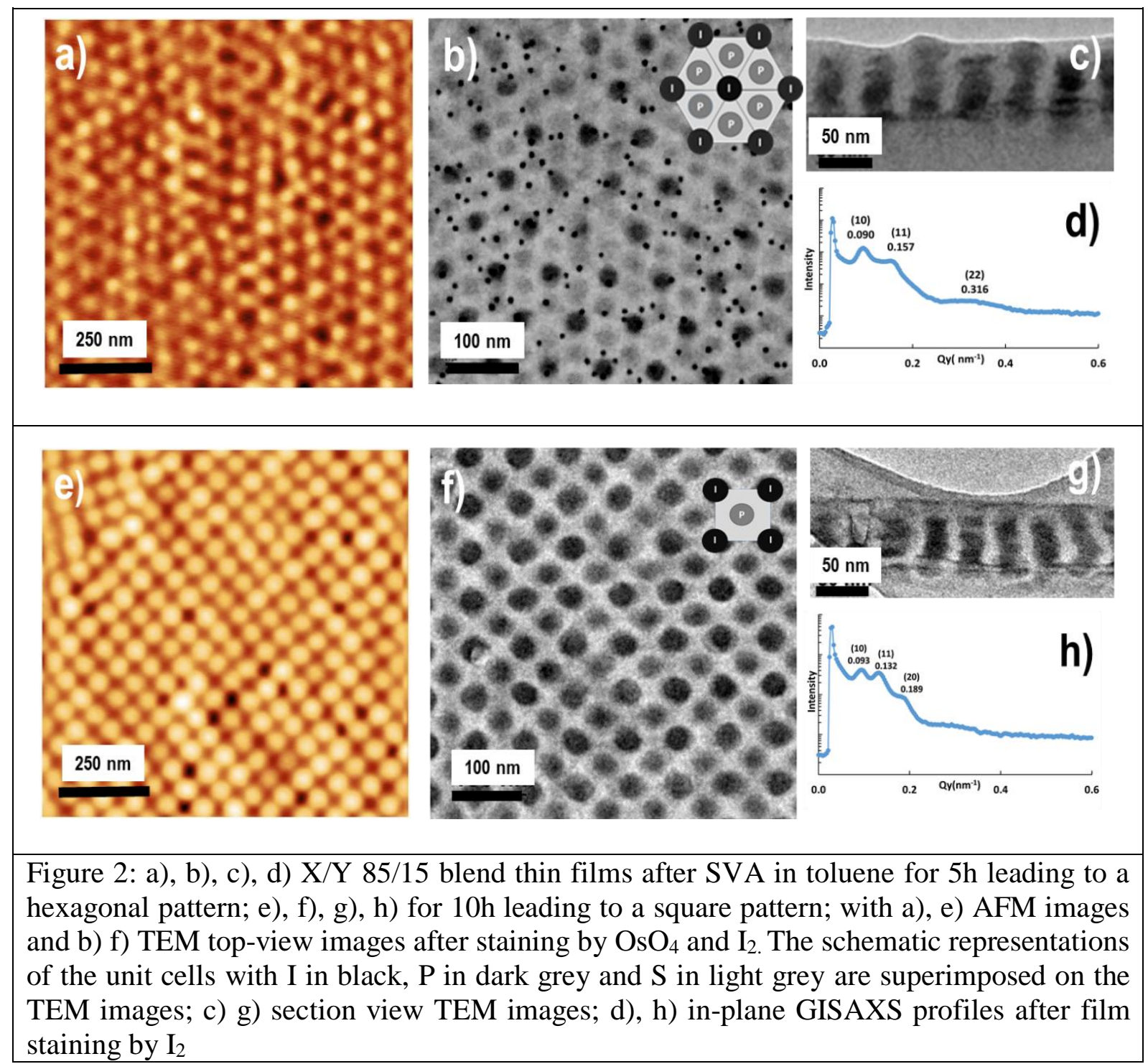

\section{X/Y 85/15 blend square pattern}

Figure 2e displays AFM image exhibiting a square pattern in the surface with a center-to-center distance between the features evaluated to $69 \mathrm{~nm}$. This is consistent with TEM top-view image obtained after selective successive staining by $\mathrm{OsO}_{4}$ and $\mathrm{I}_{2}$, presenting round black domains of I adopting a square pattern (Figure 2f). Each I domain is surrounded by 4 round dark grey domains of P. I and P networks are interwoven. The characteristic distance is $69 \mathrm{~nm}$ and I and $\mathrm{P}$ domains sized respectively 32 and $30 \mathrm{~nm}$. TEM section view exhibited rods perpendicular to the surface (Figure 2g). This is in agreement with the GISAXS pattern performed on film after 
staining by $\mathrm{I}_{2}$ presenting peaks with an extension parallel to the $\mathrm{q}_{\mathrm{z}}$ axis (see $\mathrm{SI}-2 \mathrm{~b}$ ). The in-plane profile (Figure $2 \mathrm{~h}$ ) presents two main peaks at 0.093 and $0.132 \mathrm{~nm}^{-1}$ corresponding to (10) and (11) in ratio $\sqrt{ } 2$ of a square pattern in plane with a cell unit length of $68 \mathrm{~nm}$.

\section{$\mathrm{X} / \mathrm{Y}$ 60/40 blend}

Under toluene exposure, thin film of $\mathrm{X} / \mathrm{Y} 60 / 40$ evolved through different types of morphologies in surface (displayed Figure 3 and in SI-3) starting from disorganized morphology to square pattern, then hexagonal pattern and finally to disorganized film.

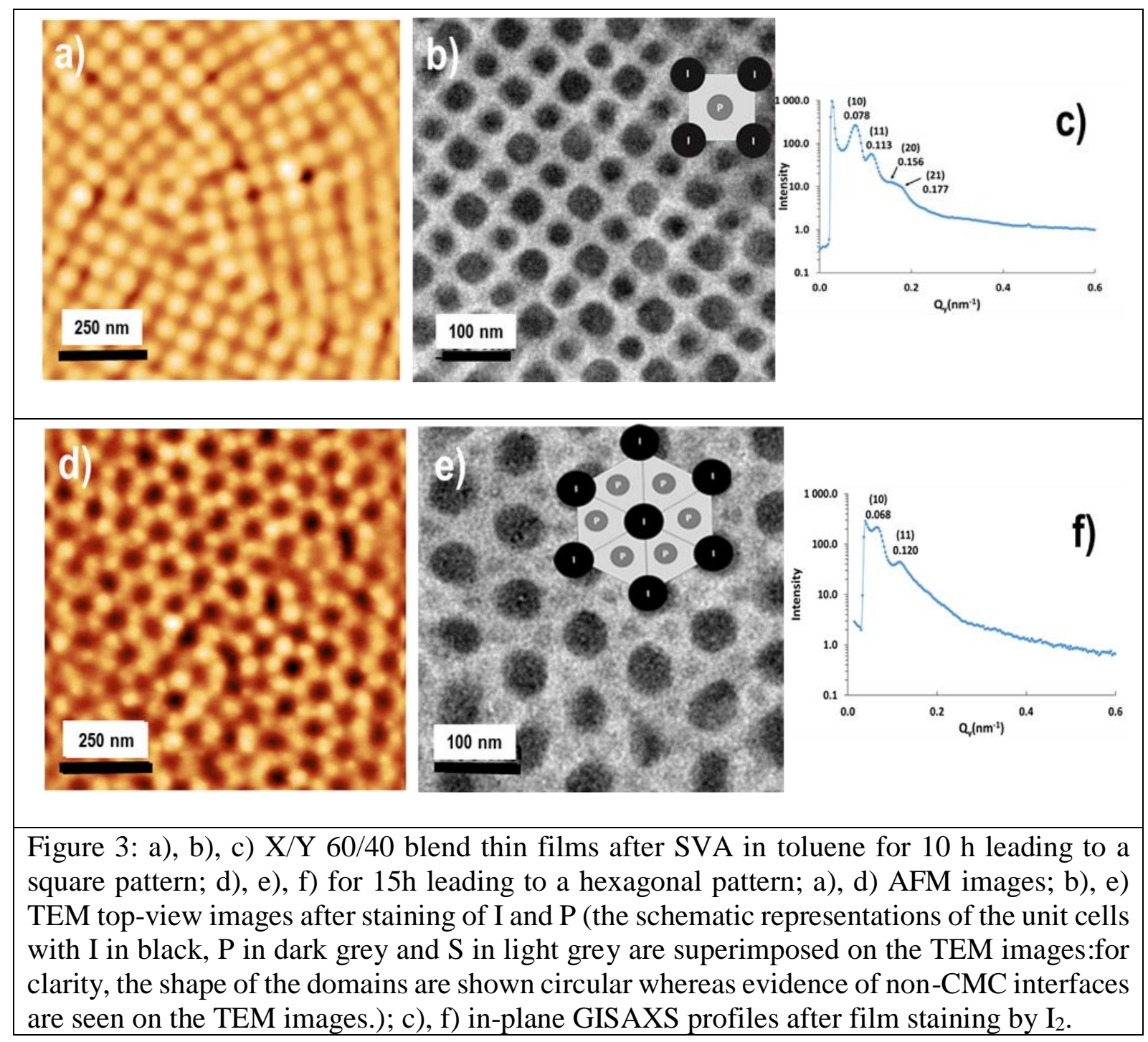




\section{X/Y 60/40 blend square pattern}

Figure 3a displays AFM image of $\mathrm{X} / \mathrm{Y}$ 60/40 film exhibiting a square pattern with a characteristic distance of $80 \mathrm{~nm}$. TEM top view image (Figure 3b), after selective successive staining by $\mathrm{OsO}_{4}$ and $\mathrm{I}_{2}$ exposures, shows two interwoven networks with a cell length of $81 \mathrm{~nm}$ of round black domains (I) and dark grey domains (P) into an unstained matrix (S). I and P domains exhibit marked difference of sizes, I domains diameter being higher than those of $\mathrm{P}$ (42 $\mathrm{nm}$ versus $33 \mathrm{~nm}$ ). The GISAXS pattern obtained on this film after $\mathrm{I}_{2}$ staining presents continuous Bragg rods with an extension parallel to the $\mathrm{q}_{\mathrm{z}}$ axis (see SI-4a) revealing the axial extension of the structure perpendicularly to the surface. The in-plane profile (Figure 3c) presents four main peaks at $0.078,0.113,0.156,0.177 \mathrm{~nm}^{-1}$ corresponding to (10), (11), (20) and (21) diffraction peaks in ratio $\sqrt{2}, 2$ and $\sqrt{5}$ to the first one, in total agreement with a tetragonal pattern with the cylinders perpendicular to the surface plane within a unit cell length of $81 \mathrm{~nm}$.

\section{X/Y 60/40 blend hexagonal pattern}

Figure 3d displays AFM image of a film exhibiting a centered hexagonal pattern of depressions, each depression being surrounded by 6 bumps hexagonally arranged. The center-to-center distance of the depression is evaluated to $95 \mathrm{~nm}$. Figure 3e displays the TEM top-view image after successive staining by $\mathrm{OsO}_{4}$ and $\mathrm{I}_{2}$ : round black domains of I adopt a centered hexagonal pattern. Each I domain is surrounded by 6 grey domains of $\mathrm{P}$. This morphology will be designed thereafter by "centered hexagons of I". The distance between I domains is evaluated to $100 \mathrm{~nm}$ and reported in table 3 . In this case, the I domains have a diameter $(50 \mathrm{~nm})$ at least twice the one of $\mathrm{P}$ domains $(25 \mathrm{~nm})$. The GISAXS pattern obtained on film after $\mathrm{I}_{2}$ staining presents peaks with an extension parallel to the $\mathrm{q}_{\mathrm{z}}$ axis (see SI-4b), signature of a homogeneity of the 
film perpendicularly to the substrate. The in-plane profile in Figure $3 f$ presents two main peaks at 0.068 and $0.120 \mathrm{~nm}^{-1}$ corresponding to (10) and (11) in ratio $\sqrt{3}$ in agreement with a hexagonal pattern in plane with a cell unit length of $91 \mathrm{~nm}$.

\section{Transition pathway from hexagonal towards tetragonal pattern and vice-versa}

Before discussing the origin of the ordered phases, one has to note that both blends reach a disordered state for longer annealing time $(>20 \mathrm{~h})$, with no evidence of short and long range order. This suggests that an order-disorder transition was reached during SVA, resulting from a major swelling degree (the fast evaporation freezes the system into a more or less disordered state). This suggests that the morphologies we will now discuss are not at equilibrium, but represent kinetically defined transitory structures.

For the two blends, Table 3 summarizes the characteristics of the morphologies in bulk (obtained from previous work [14]) and in thin films (obtained in this work). In thin films, the characteristic distances measured in AFM, TEM and GISAXS are all in well agreement and allow to calculate the apparent composition of each phase (I, S and P) based on simple geometric considerations (see SI-5). The overall composition of the block copolymer in the film being constant, the variation of the apparent volume fraction determined by TEM can only be explained by a selective migration of one block towards the interface, forming a uniform wetting layer (non-observable on a top-view TEM micrograph) and depleting the inner portion of the film from this block. In turn, this will modify the morphology, as we will explain below.

\begin{tabular}{|c|c|c|c|c|c|c|}
\hline blend & \multicolumn{3}{|c|}{$\mathrm{X} / \mathrm{Y} 85 / 15$} & \multicolumn{3}{|c|}{$\mathrm{X} / \mathrm{Y} 60 / 40$} \\
\hline \multirow{2}{*}{$\begin{array}{l}\text { observed } \\
\text { structure }\end{array}$} & bulk [14] & $\begin{array}{l}\text { SVA, } \\
\text { toluene, } \\
5 \mathrm{~h}\end{array}$ & $\begin{array}{l}\text { SVA, } \\
\text { toluene, } \\
10 \mathrm{~h}\end{array}$ & bulk [14] & $\begin{array}{l}\text { SVA, } \\
\text { toluene, } \\
10 \mathrm{~h}\end{array}$ & $\begin{array}{c}\text { SVA, } \\
\text { toluene, } \\
15 \mathrm{~h}\end{array}$ \\
\hline & $\begin{array}{ll}(1) & 1 \\
(1) & 1\end{array}$ & $\begin{array}{lllll}1 & P & 1 \\
1 & P & P \\
1 & P & P \\
1 & P & 1 \\
\end{array}$ & $\begin{array}{l}\text { (1) } 1 \\
\text { (1) } 1\end{array}$ & & (1) & $e_{1}^{1} \cdot 1$ \\
\hline
\end{tabular}




\begin{tabular}{|c|c|c|c|c|c|c|c|}
\hline AFM & $\mathrm{d}_{\mathrm{cc}}$ & & 72 & 69 & & 80 & 95 \\
\hline \multirow{2}{*}{$\mathrm{TEM}$} & $\mathrm{d}_{\mathrm{cc}}$ & $73^{*}$ & 77 & 69 & $87^{*}$ & 81 & 100 \\
& $\mathrm{D}_{\mathrm{I}}$ & $34^{*}$ & 27 & 32 & $50^{*}$ & 42 & 50 \\
& $\mathrm{DP}_{\mathrm{P}}$ & $34^{*}$ & 28 & 30 & $34^{*}$ & 33 & 25 \\
\hline GISAXS & $\mathrm{d}_{\mathrm{cc}}$ & & 70 & 68 & & 81 & 91 \\
\hline \multicolumn{2}{c|}{$\begin{array}{c}\text { I:S:P Parent } \\
\text { composition }\end{array}$} & $0.17: 0.66: 0.17$ & $0.11: 0.65: 0.24$ & $0.17: 0.68: 0.15$ & $0.26: 0.62: 0.12$ & $0.21: 0.66: 0.13$ & $0.23: 0.66: 0.11$ \\
\hline
\end{tabular}

Table 3: Characteristic distances (in $\mathrm{nm}$ ) of the different observed patterns for $\mathrm{X} / \mathrm{Y} 85 / 15$, $\mathrm{X} / \mathrm{Y} 60 / 40 ; \mathrm{d}_{\mathrm{cc}}$ center to center distance for the cylinders of the centered hexagon or center to center distance of I (P) cylinders in the square pattern ; D cylinder diameter. For clarity, the shapes of all domains are represented by circular features, which may not reflect the actual geometry (see for example Fig. 5). (I:S:P) volume fraction of the bulk and observed patterns in thin film (I in yellow, $\mathrm{S}$ in grey and $\mathrm{P}$ in blue). *In bulk, the composition of the observed patterns is the same that the blend composition. In thin film, the given composition is determined from the TEM distances (apparent composition).

\section{X/Y 85/15 blend}

The X/Y 85/15 blend with a (I:S:P) native volume fraction of $(0.17: 0.66: 0.17)$ was previously studied by Asai [14]. In good agreement with predictions (volume fraction of the middle-block between 0.60 and 0.66 and equal volume fractions of both end-blocks), this blend exhibited in the bulk a tetragonal morphology. The cylinders of I and P have the same size $(34 \mathrm{~nm})$ as schematized in table 3 . In the thin film version of this blend, the tetragonal morphology transitioned to an intermediate double hexagonal structure in the early stage of the toluene vapor annealing, with a modification of its apparent composition (0.11:0.65:0.24). This composition is depleted in I compared to the initial one (0.17:0.66:0.17). This could be explained by the tendency of I to segregate to the surface and to form a wetting layer at the interface film/air [22, 23] due its lowest surface energy. The surface energies of I, S, and P were herein determined on homopolymer films using contact angle measurements of different liquids and were estimated to 32,40 and $47 \mathrm{~mJ} . \mathrm{m}^{-2}$ for I, S and P in agreement with the literature $[23,24]$. The I segregation at the interface film/air consequently induces an inner composition of the films with a lack of I. 
We observed centered hexagons of I cylinders (surrounded by P cylinders) in this work, whereas Asai obtained centered hexagons of P cylinders (surrounded by I cylinders) for the linear triblock terpolymer X (I:S:P) with a similar composition $(0.12: 0.67: 0.21)$ [14]. To explain these discrepancies, the stabilities of these two double hexagonal morphologies (centered hexagons of P versus centered hexagons of I) must be considered. As $\chi_{\text {S-P }}$ is higher than $\chi_{\text {S-I }}$ $\left(\chi_{\mathrm{S}-\mathrm{I}}=0.06 ; \chi_{\mathrm{S}-\mathrm{P}}=0.1 ; \chi_{\mathrm{I}-\mathrm{P}}=0.2[25]\right)$, the system tends to limit the amount of P/S interface compared to the I/S interface and thus to increase the ratio (Ir) between the I/S interface area and P/S interface area. From the interfacial energy standpoint, the most favorable system corresponds to centered hexagon of P (highest value of Ir in the figure 4a in good accordance with the morphologies reported literature. $[14,15]$

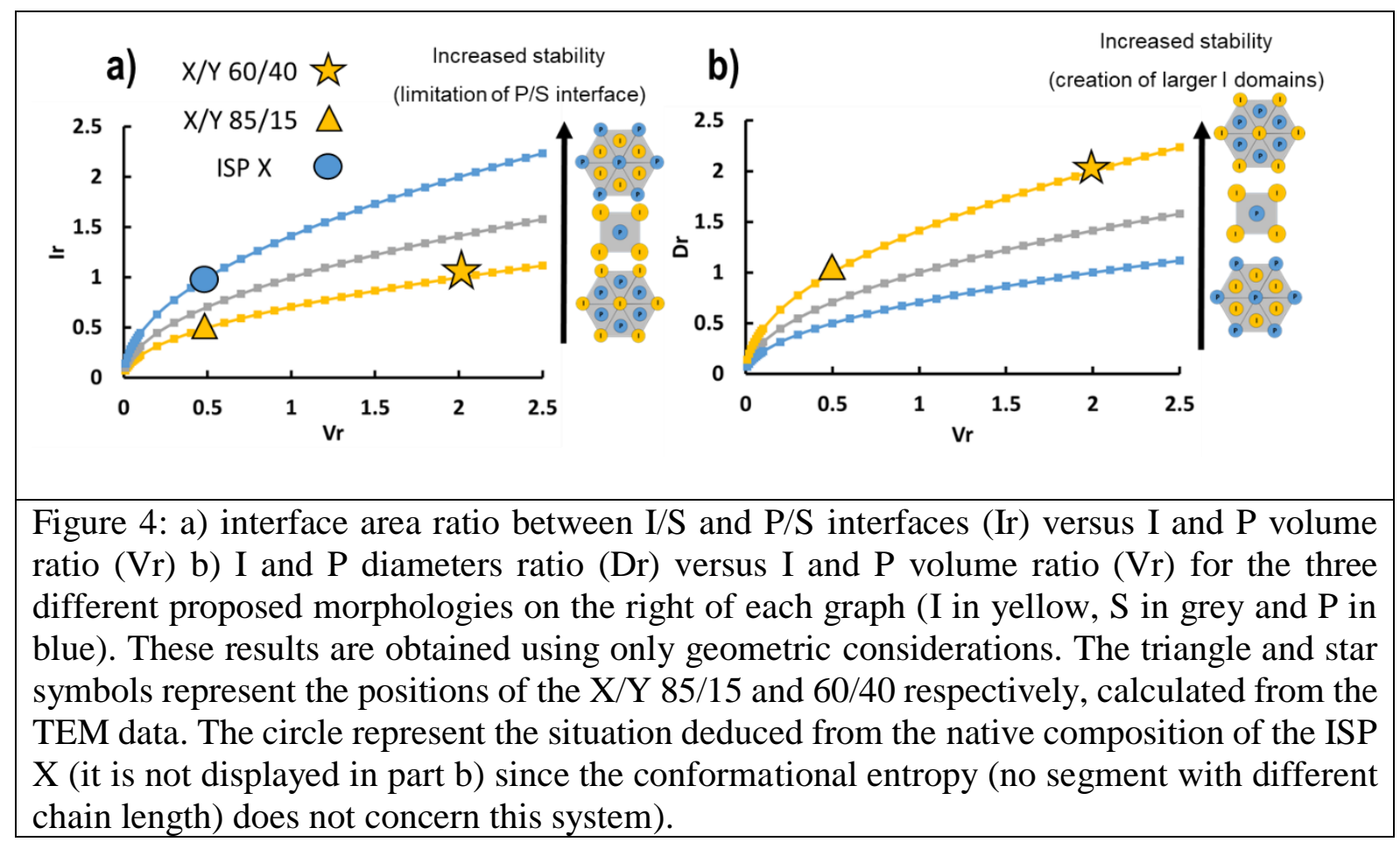

In our case, we obtained centered hexagons of I, which corresponds to the less favorable system from the interfacial energy standpoint. This can be seen in Figure 4a since the Ir values corresponding to the centered hexagons of I are below the values corresponding to the centered 
hexagons of $\mathrm{P}$ (the red triangle and star points represent the positions of the $\mathrm{X} / \mathrm{Y} 85 / 15$ and 60/40 respectively, calculated from the TEM data). However, chains with different sizes are present in each cylindrical domain since the studied system is a blend of two block copolymers with different compositions. In the X/Y studied blends, we mixed ISP X (I 357 and P 625 repeating units) with ISP Y (I 1649 and P 72 repeating units). In this X/Y 85/15 blend, I domains contain both chains of 357 units (85\%) and chains of 1649 units (15\%). Consequently, we suggest that the system tends to adopt I domains as large as possible because it favors the location of the longest chains in the largest domains, to keep their conformational entropy loss as small as possible. The double hexagonal morphology with centered hexagon of I gives larger diameter for the I domains than for $\mathrm{P}$ domains (highest value of ratio between the $\mathrm{I}$ and $\mathrm{P}$ diameters (Dr) as in Figure 4b. It can be suggested that this "size effect" overcomes the P/S interface minimization, favoring the formation of a double hexagonal morphology with centered hexagon of I. Very interestingly, these results suggest that the presence of a moderate amount of long I chains (15\%) induces an inversion of morphology, from centered hexagons of P to centered hexagons of I. For comparison purpose, in the ISP X case [14] with a composition of (0.12:0.67:0.21) and centered hexagons of P, I has 357 units and P 625 units: the shortest chains locates in the smallest domains of I and the longest ones in the biggest ones (P domains). The diameter of I domains is half the diameter of $\mathrm{P}$ domains. In this case, the interfacial stability is in good accordance with entropic considerations since the size of the domains are consistent with the size of the chains.

For longer annealing time, the segregation of $\mathrm{P}$ at the interface substrate/film occurs [22], lowering the proportion of $\mathrm{P}$ in the inner film, which compensates for the depletion of I that previously occurred in the first stages of the annealing. $\mathrm{P}$ is also prone to migration (towards the substrate interface (the surface energy of the substrate being estimated to $46 \mathrm{~mJ} . \mathrm{m}^{-2}$ [22] ) but because I swells more, and faster than P2VP (as shown in SI-6), the formation of the wetting 
layer of I will occur first. The apparent composition value at that stage (extracted from the TEM) is now symmetric $(0.17: 0.68: 0.15)$ and very close to the initial composition and drives the formation of the native tetragonal morphology. Note that the domains have similar sizes between hexagonal and tetragonal structures. This is in agreement with the fact that the size of the domains is imposed by the size of the blocks and not the volume fraction in the blend.

\section{X/Y 60/40 blend}

In the case of $\mathrm{X} / \mathrm{Y} 60 / 40$ blend, the same interpretation of the morphological evolution upon annealing can be given. The initial bulk volume fraction (I:S:P) is $(0.26: 0.62: 0.12)$. Despite a composition that would normally form a hexagonal pattern, a tetragonal morphology is formed in bulk, with interwoven tetragonal arrays of I and P cylinders, the domains of I being much larger $(50 \mathrm{~nm})$ than those of $\mathrm{P}(34 \mathrm{~nm})[14]$. This is again due to the size of I and P chains. I domains contains $40 \%$ of chains of 1649 units whereas the maximal chain length in the P domains is only 625 units. In this case, the formation of a tetragonal pattern corresponds to a good balance between the limitation of the P/S interface (moderate value of Ir in Fig 4a) and formation of I domains large enough to accommodate the longer I chains (intermediate values of Dr in Fig 4b).

During the first stage of annealing, I segregation at the surface occurs first, which in turn reduces the amount of I in the inner structure. However, this reduction lets the system with a composition (I:S:P) (0.21:0.66:0.13) depleted in I but still within the range of a tetragonal structure. Note that as previously, the I domains size $(42 \mathrm{~nm})$ is gouverned by the size of long chains of I, which in this case represents $40 \%$ of long chains.

Further solvent exposure led to $\mathrm{P}$ segregation at the interface film/substrate and enhanced proportion of $I$ in the inner films projecting the system $(0.23: 0.66: 0.11)$ outside the area of 
tetragonal structure, into the area of hexagonal one. Then again, the size of I domains is imposed by the chains length and this leads to centered hexagons of I surrounded by domains of P. Thin film stabilizes this structure although the composition is the same of the bulk which exhibits a tetragonal pattern. This phenomenon has been already observed for an ISP terpolymer which exhibits in bulk a gyroid pattern and in thin film evolves to a tetragonal morphology [25].

In order to verify the central role of the length of the I chains in the formation of the morphology, we considered a blend of a ISP triblock and IS diblock with a (I:S:P) volume proportion of $(0.20: 0.59: 0.21)$ in which both $\mathrm{I}$ and $\mathrm{P}$ chains are within the same range of repeating units number. This time, as show in SI-7, this blend led in thin film to a cylindrical morphology with centered hexagon (with a characteristic distance of $84 \mathrm{~nm}$ ) of cylinders of $\mathrm{P}$ (diameter of $36 \mathrm{~nm}$ ) surrounded by cylinders of I with a diameter of $27 \mathrm{~nm}$ leading to an (I:S:P) apparent composition (0.18:0.65:0.17). In this case, the interface area of $\mathrm{P} / \mathrm{S}$ is less than the interface area of $\mathrm{I} / \mathrm{S}$ (Interface ratio $=1.5$ ). The diameter ratio of the I and $\mathrm{P}$ domains is 0.75 , compatible with the block lengths of I and P (59\% with 96 units and $41 \%$ with 576 units for I chains; P domains contain chains of 513 units). This confirms the central role of the long chains of I in the inverted morphology observed previously. The presence of IS diblock allowing S to fill the space between the I domains is another favorable factor to observe this morphology.

Another point that has to be discussed is the shape of domains. It was demonstrated in previous works, that blending block copolymers exhibiting a bimodal distribution leads to non constant mean curvature (non-CMC) interface, e.g the domains are no more cylindrical but present flat interfaces [26]. This is particularly visible for the square pattern of $\mathrm{X} / \mathrm{Y} 60 / 40$, I domains have clearly a square base as shown in figure $5 \mathrm{a}$. The location of the blocks is proposed in figure $5 \mathrm{~b}$. This example is the most striking one in this study, but for all domains with a bimodal 
distribution of chains, the different locations of the blocks and the packing frustration impose the shape of the domains.
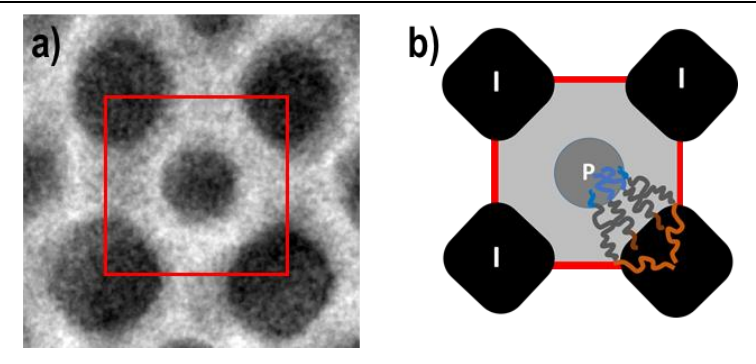

Figure 5: a) 150*150 nm TEM image of square pattern for X/Y 60/40 thin film (I in black, $\mathrm{S}$ in grey and $\mathrm{P}$ in dark grey) and schematic representation

\section{Conclusion}

The morphology of blends of ISP linear block copolymer was investigated in thin film upon solvent vapor annealing. Unreported morphologies were obtained, with marked differences compared to the bulk morphology. A double hexagonal morphology was formed, with centered hexagons of I and surrounding hexagons of $\mathrm{P}$, which is not predicted nor observed for neat block copolymer samples. In this thin film configuration, the formation of non-synchronized wetting layers of I and P leads to modification of the inner composition of the film, driving the observed transitions. Although the system must adopt the lowest P/S interface, our results suggest that the presence of long chains (here the I chains) is playing a major role, favoring the formation of I domains with bigger size, in order to keep their conformation entropy loss as small as possible. 


\section{Acknowledgements}

We gratefully acknowledge the financial support provided to the PIVOTS project by the Région Centre - Val de Loire (ARD 2020 program and CPER 2015 -2020) and the French Ministry of Higher Education and Research (CPER 2015 -2020 and public service subsidy to CNRS and Université d'Orléans). Frederic Mahut is deeply acknowledged for his experimental contribution. Pascal Andreazza and Marie-Pierre Faugère are thanked for fruitful discussions and contribution to the GISAXS experiments.

\section{Data availability}

The raw/processed data required to reproduce these findings cannot be shared at this time due to technical or time limitations. 


\section{References}

[1] N. Hadjichristidis, H. Iatrou, M. Pitsikalis, S. Pispas, A. Avgeropoulos, Linear and non-linear triblock terpolymers. Synthesis, self-assembly in selective solvents and in bulk, Prog. Polym. Sci. 30 (2005) 725-782.

https://doi.org/10.1016/j.progpolymsci.2005.04.001

[2 ] J. Meuler, M. A. Hillmyer, F. S. Bates, Ordered Network Mesostructures in Block Polymer Materials Macromolecules 42 (2009) 7221-7250.https://doi.org/ 10.1021/ma9009593

[3] C. M. Bates, F. S. Bates, 50th Anniversary Perspective: Block Polymers Pure Potential Macromolecules, 50 (2017) 3-22. https://doi.org/ 10.1021/acs.macromol.6b02355

[4] C. A. Tyler, J. Qin, F. S. Bates, D. C. Morse, SCFT Study of Nonfrustrated ABC Triblock Copolymer Melts, Macromolecules 40 (2007) 4654-4668.

https://doi.org/10.1021/ma062778w

[5] U. Nagpal, François A. Detcheverry, P. F. Nealey, J. J. de Pablo, Morphologies of Linear Triblock Copolymers from Monte Carlo Simulations, Macromolecules 44 (2011) 5490-5497. https://doi.org/ 10.1021/ma200330f

[6] J. Qin, F. S. Bates, D.C. Morse, Phase Behavior of Nonfrustrated ABC Triblock Copolymers: Weak and Intermediate Segregation, Macromolecules 43 (2010) 5128-5136. https://doi.org/ 10.1021/ma100400q

[7] S. Phan, G. H. Fredrickson, Morphology of Symmetric ABC Triblock Copolymers in the Strong Segregation Limit, Macromolecules 31 (1998) 59-63.

https://doi.org/10.1021/ma971046o 
[8] W. Zheng, Z.-G. Wang, Morphology of ABC Triblock Copolymers Macromolecules 28 (1995) 7215-7223. https://doi.org/10.1021/ma00125a026

[9] J. Chatterjee, S. Jain, F. S. Bates, Comprehensive Phase Behavior of Poly(isoprene-bstyrene-b-ethylene oxide) Triblock Copolymers Macromolecules 40 (2007) 2882-2896. https://doi.org/ 10.1021/ma062249s

[10] M. S. Tureau, W.-F. Kuan, L. Rong, B. S. Hsiao, T. H. Epps III, Inducing Order from Disordered Copolymers: On Demand Generation of Triblock Morphologies Including Networks, Macromolecules 45 (2012) 4599-4605 https://doi.org/10.1021/ma300365h.

[11] M. S. Tureau, L. Rong, B. S. Hsiao, T. H. Epps, Phase behavior of neat triblock copolymers and copolymer/homopolymer blends near network phase windows, Macromolecules 43 (2010) 9039-9048. https://doi.org/10.1021/ma100783y.

[12] Y. Mogi, M. Nomura, H. Kotsuji, K. Ohnishi, Y. Matsushita, I. Noda, Superlattice Structures in Morphologies of the ABC Triblock Copolymers, Macromolecules 27 ( 1994) 6755-6760. https://doi.org/10.1021/ma00101a013

[13] S. Brinkmann, R. Stadler, E. L. Thomas, New Structural Motif in Hexagonally Ordered Cylindrical Ternary (ABC) Block Copolymer Microdomains, Macromolecules 31(1998) 65666572. https://doi.org/10.1021/ma980103q

[14] Y. Asai, A. Takano, Y. Matsushita, Asymmetric double tetragonal domain packing from ABC triblock terpolymer blends with chain length difference, MacromoleculesMacromol. 49 (2016) 6940-6946. https://doi.org/ 10.1021/acs.macromol.6b01670

[15] Y. Izumi, M. Yamada, A. Takano, Y. Matsushita, A New Periodic Pattern with FiveNeighbored Domain Packing from ABC Triblock Terpolymer/B Homopolymer Blend J. Polym. Sci., Part B: Polym. Phys. 53 (2015) 907-91. https://doi.org/10.1002/polb.23714 
[16] Y. Asai, K. Yamada, M. Yamada, A. Takano, Y. Matsushita, Formation of TetragonallyPacked Rectangular Cylinders from ABC Block Terpolymer Blends, ACS Macro Lett. 3 (2014) 166-169. https://doi.org/10.1021/mz400647v

[17] Y. Asai, A. Takano, Y. Matsushita, Creation of Cylindrical Morphologies with Extremely Large Oblong Unit Lattices from ABC Block Terpolymer Blends, Macromolecules 48 (2015) 1538-1542. https://doi.org/10.1021/ma5025818

[18] C. Sinturel, M. Vayer, M. Morris, M. A. Hillmyer, Solvent Vapor Annealing of Block Polymer Thin films, Macromolecules $46 \quad$ (2013) 5399-5415. https://doi.org/10.1021/ma400735a

[19] V. P. Chuang, J. Gwyther, R. A. Mickiewicz, I. Manners, C.A. Ross, Templated SelfAssembly of Square Symmetry Arrays from an ABC Triblock Terpolymer, Nanolett. 9 (2009) 4364-4369. https://doi.org/ 10.1021/n1902646e

[20] A. Guliyeva, M. Vayer, F. Warmont, A.-M. Faugère, P. Andreazza, A. Takano, Y.Matsushita, C.Sinturel, Thin Films with Perpendicular Tetragonally Packed Rectangular Rods Obtained from Blends of Linear ABC Block Terpolymers, ACS Macro Lett., 7 ( 2018) 789 - 794. https://doi.org/ 10.1021/acsmacrolett.8b00272

[21] Y. Mogi, H. Kotsuji , Y. Kaneko , K. Mori, Y. Matsushita, I. Noda, Preparation and Morphology of Triblock Copolymers of the ABC Type, Macromolecules 25 (1992) 5408-5411. https://doi.org/10.1021/ma00046a043

[22] A. Guliyeva, M. Vayer, F. Warmont, A. Takano, Y. Matsushita, C. Sinturel, Transition Pathway Between Gyroid and Cylindrical Morphology in Linear Triblock Terpolymer Thin Films. Macromolecules 52 (2019) 6641-6644. https://doi.org/10.1021/acs.macromol.9b01263 
[23] J. G. Son, J. Gwyther, J.-B. Chang, K. K. Berggren, I. Manners, C. A. Ross, Highly Ordered Square Arrays from a Templated ABC Triblock Terpolymer, Nano Lett. 11 (2011) 2849-2855. https://doi.org/10.1021/nl201262f

[24] J. Zhang, M. B. Clark, C. Wu, M. Li, P. Trefonas, P. D. Hustad, Orientation Control in Thin Films of a High- $\chi$ Block Copolymer with a Surface Active Embedded Neutral Layer, Nano Lett. 16 (2016) 728-735. https://doi.org/10.1021/acs.nanolett.5b04602

[25] S. Ahn, J. Kwak, C. Choi, Y. Seo, J. K. Kim, Gyroid structures at highly asymmetric volume fractions by blending of $\mathrm{ABC}$ triblock terpolymer and $\mathrm{AB}$ diblock copolymer Macromolecules 50 (2017) 9008-9014. https://doi.org/10.1021/acs.macromol.7b01734

[26] Y. Matsushita, A. Takano, M. Vayer, C. Sinturel, Non-classical block-copolymer selfassembly resulting from a constrained location of chains and junctions, Adv. Mater. Interf. (2020) 1902007. https://doi.org/10.1002/admi.201902007 